V. M. SOTO HERMOSO*

P. GERONA GALDAME**

M. GUTIÉRREZ DÁVILA*

L. P. RODRÍGUEZ RODRÍGUEZ**

* Laboratorio de Biomecánica del Departamento de Educación Física y Deportiva de la Universidad de Granada.

** Departamento de Medicina Física y de Rehabilitación de la Facultad de Medicina de la Universidad

Complutense de Madrid.

\section{Desarrollo de un sistema fotogramétrico tridimensional computerizado no invasivo para el análisis de la columna vertebral humana}

Development of a non-invasive
computerized three-dimensional
photogrammetric system for
analyzing the human spine

\section{Resumen}

El presente trabajo expone el desarrollo de un sistema fotogramétrico tridimensional (3D) no invasivo basado en la utilización de imágenes radiológicas que permite el análisis de la columna vertebral humana para su aplicación en el ámbito de la investigación básica acerca del comportamiento de la columna y su utilización práctica para facilitar el diagnóstico clínico en los centros médicos. A partir de la información obtenida mediante imágenes radiológicas son aplicadas técnicas fotogramétricas $3 D$ computerizadas que permiten con una adecuada metodología no invasiva la obtención de diversos parámetros biomecánicos que permiten determinar el comportamiento estático y dinámico de la columna. Asimismo se desarrollan ciertos algoritmos de representación gráfica tridimensional de la columna vertebral humana que la simulan de forma realista; esto puede suponer un avance importante para su utilización en el perfeccionamiento del diagnóstico clínico. El sistema desarrollado ha sido aplicado de forma práctica sobre una muestra de pacientes afectados por diversos grados de escoliosis.

Palabras clave: Biomecánica. Columna vertebral. Fotogrametría tridimensional.

\begin{abstract}
Summary
A non-invasive three-dimensional (3D) photogrammetric system was developed that was based on using radiographic images for the analysis of the human spine in basic research of spinal behavior and in practical diagnostic applications in medical centers. Using information obtained from radiographic images, computerized $3 D$ photogrammetric techniques were applied to obtain various biomechanical parameters for evaluating the static and dynamic behavior of the spine. Algorithms were developed for the graphic three-dimensional representation of the human spine that provide a realistically simulation, which is an important advanced for perfecting clinical diagnosis. The system was used to study a sample of patients wit different degree of scoliosis
\end{abstract}

Key words: Biomechanics. Spine. Three-dimensional photogrammetry.

\section{Introducción}

El estudio de la columna vertebral implica unos beneficios claros e inmediatos sobre la salud y calidad de vida

\footnotetext{
Correspondencia:

V. M. SOTO HERMOSO. Laboratorio de Biomecánica del Departamento de Educación Física y Deportiva de la Universidad de Granada.
}

del ser humano, ya que ésta es una de las estructuras anatómicas fundamentales del organismo humano que interviene en funciones tan variadas como desplazamientos, situaciones estáticas, sostén de órganos, transmisión de fuerzas, reducción de los estrés más habituales, etc. De esta forma queda manifiestamente claro que el estudio del comportamiento de la columna es una de las áreas fundamentales de investigación básica y aplicada. 
La biomecánica y la medicina física y de rehabilitación han sido algunas de las disciplinas que se han interesado y profundizado en el estudio del comportamiento de la columna vertebral, ya sea en situaciones normales como patológicas. La medicina física y de rehabilitación permite profundizar en aspectos relacionados con las técnicas terapéuticas necesarias para cada patología, tratamientos ortopédicos, electroterapia, cinesiterapia, hidroterapia, etc. Desde el punto de vista de la biomecánica es posible el análisis de las fuerzas externas e internas que afectan a la estructura vertebral, ergonomía postural estática y dinámica, mecánica estructural de los tejidos, así como su comportamiento ante los estrés generados, análisis cinemático y dinámico, etc.

La investigación moderna aplicada al análisis de la columna requiere la adaptación de equipos e investigadores a las nuevas tecnologías informatizadas que permitan y faciliten la labor del investigador. El uso del ordenador se hace imprescindible en toda tecnología que pretenda ser rigurosa y precisa; asimismo, también facilita la labor automatizando procesos, incrementando la velocidad de cálculo, incrementando la precisión de la medida, etc. ${ }^{13}$. Esto supone un esfuerzo humano para adaptarse a los sistemas, así como un coste considerable para los organismos, ya que en la mayoría de los casos se opta por la adquisición de costosos sistemas que no suelen resolver las necesidades específicas que cada investigación particular requiere. En muchos casos se opta por el desarrollo de tecnologías propias de uso restringido para investigaciones muy específicas que no suelen estar disponibles en otros centros.

El sistema elaborado desarrolla una tecnología que facilita el análisis del comportamiento de la columna vertebral, utilizando para ello una tecnología no invasiva de coste relativamente reducido, adaptable y flexible a las necesidades de diversos tipos de situaciones e investigaciones. Para ello es imprescindible un soporte informatizado que pueda ser aplicado no sólo con vistas a la investigación básica en centros universitarios, sino también en centros de diagnóstico y asistencia clínica.

El punto de partida básico de un sistema tecnológico de análisis de la columna parte del condicionante de la tecnología medidora utilizada, ésta a su vez podría clasificarse en las siguientes alternativas: a) radiología convencional, b) radiología digital; c) mielografía; d) tomografía computerizada (escáner); e) ultrasonografía, y f) resonancia magnética.

La radiología es en la actualidad el método más extendido universalmente y representa la base para el seguimiento del comportamiento del raquis, aunque existen otras múltiples técnicas, algunas de ellas en fase de desarrollo y perfeccionamiento (técnicas tomográficas, ultrasónicas, radiología digital...) que han quedado la mayoría de las veces como aportaciones a la investigación, pero no se han aplicado clínicamente a gran escala.

Las limitaciones inherentes en la utilización de información bidimensional (2D), tal y como se plantea al utilizar uná única imagen radiológica, limita la precisión de los estudios, ya que la información puede no ser completa. Es necesario, pues, que se implementen sistemas que faciliten el análisis tridimensional del raquis. Con la llegada de las nuevas tecnologías de tratamiento de imagen basadas en la utilización del ordenador actualmente es posible obtener información espacial que sea plasmada en representaciones gráficas tridimensionales (3D) de la columna vertebral de forma indirecta sin necesidad de cirugía u otras técnicas invasivas.

Desde el inicio de la década de los ochenta han ido apareciendo diversas publicaciones de autores tales como DE SMET y cols. ${ }^{4,5,6,7}$, DE GIORGI y cols. ${ }^{3}$, etc., en las cuales se proponen diferentes métodos para el análisis de deformidades, basados en imágenes radiográficas, que aportan información gráfica 3D de la columna vertebral analizada. Otros autores como PEARCY y cols. ${ }^{12}$ han estudiado la influencia del movimiento de deambulación sobre la columna utilizando técnicas 3D. A los estudios iniciales previamente citados se añaden múltiples publicaciones hasta la actualidad: STOKES y cols. ${ }^{14,15}, 16$, HOWEL y DICKSON $^{9}$, KOJIMA y KUROKAWA ${ }^{10,11}$, DEACON y cols. ${ }^{2}$, GRAFT y cols. ${ }^{8}$, etc.

La colaboración planteada entre investigadores del Laboratorio de Biomecánica del Departamento de Educación Física y Deportiva de la Universidad de Granada y del Departamento de Medicina Física y de Rehabilitación de la Facultad de Medicina de la Universidad Complutense de Madrid ha dado como fruto el desarrollo y perfeccionamiento de tecnologías y sistemas fotogramétricos tridimensionales que facilitan el estudio de la columna vertebral.

\section{Método}

\section{Qbietivos}

En la elaboración de este trabajo se han planteado una serie de objetivos de desarrollo metodológico y tecnológico, así como la implementación de soportes lógicos (software) que regulen, automaticen y controlen de forma rigurosa el proceso de obtención de datos, tratamiento y exposición de resultados utilizando técnicas 3D. De forma resumida se han conseguido los siguientes objetivos:

- Crear una metodología basada en técnicas fotogramétricas tridimensionales no invasivas mediante la utilización de imágenes radiológicas que permiten la obtención de parámetros espaciales de la columna vertebral humana.

- Desarrollar algoritmos que permitan la creación de imágenes gráficas realistas de la columna vertebral humana teniendo en cuenta los parámetros antropométricos específicos de los sujetos analizados.

- Ajustar y validar los sistemas desarrollados con diversos estudios aplicados con poblaciones afectadas por escoliosis.

\section{Metodología fotogramétrica tridimensional}

Desde el punto de vista de la metodología de análisis y la tecnología a utilizar se han seleccionado las técnicas fo- 


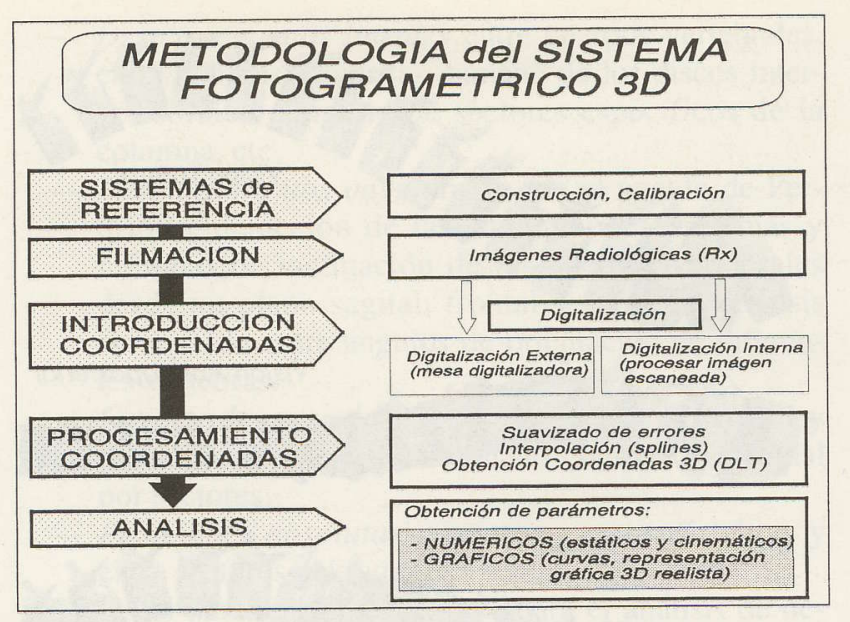

FIG. 1.-Fases secuenciales de la metodología fotogramétrica 3D empleada.

togramétricas tridimensionales, ya que su carácter no invasivo es considerado como factor primordial en la realización de este tipo de análisis.

La metodología general del sistema fotogramétrico a utilizar es expuesta de forma esquemática en la figura 1. Este proceso consta de una serie de fases secuenciales, siendo la primera de ellas la construcción y calibración de sistemas de referencias tridimensionales necesarios para el correcto funcionamiento del algoritmo DLT utilizado en la reconstrucción espacial de las coordenadas. El objeto de referencia deberá colocarse en los límites espaciales del volumen que puede captar la placa radiológica $(\mathrm{Rx})$, de forma que no afecte al sujeto sobre el cual se realizará el análisis de su columna vertebral. Se obtendrán 2 imágenes radiológicas $(\mathrm{Rx})$ desde 2 perspectivas distintas $\left(90^{\circ}\right)$ del sujeto analizado en la posición designada por el investigador (habitualmente en posición anatómica). Para la obtención de las 2 imágenes radiológicas se utilizará una plataforma rotatoria sobre la cual se coloca el sujeto experimental y el objeto de referencia, la sucesiva rotación de la plataforma permitirá la obtención de 2 imágenes desde diferentes puntos de vista del sujeto y el objeto de referencia.

La información gráfica obtenida a partir de las 2 imágenes radiológicas deberá ser introducida dentro del ordenador para su posterior procesamiento y obtención de resultados. Esta introducción, habitualmente denominada como fase de digitalización, podrá ser realizada mediante 2 tipos de tecnologías: a) digitalización externa en una mesa digitalizadora, o bien b) digitalización interna procesando las imágenes radiológicas previamente escaneadas dentro del ordenador.

La posición de las vértebras será determinada a partir de la digitalización del centro geométrico de los discos intervertebrales, los cuales son claros indicadores de la posición de los cuerpos vertebrales. Estas coordenadas o parámetros bidimensionales (2D) obtenidos a partir de la digitalización definen una base de datos que caracteriza la morfología de la columna vertebral analizada.
Los periféricos utilizados en la fase de digitalización son controlados por un ordenador PC-compatible en el cual se almacenarán las bases de datos de coordenadas para la posterior fase de tratamiento. Una vez introducida en el ordenador la base de datos de la columna analizada, estos parámetros pasarán por una serie de fases intermedias durante las cuales serán transformadas y alteradas hasta la obtención de las definitivas coordenadas espaciales (3D), sobre las cuales se realizará el proceso de obtención de resultados. La primera fase de este procesamiento de las coordenadas realizará un suavizado o minimización de los posibles errores aleatorios y/o sistemáticos que hayan podido generarse durante la fase de digitalización. En una segunda fase es necesaria la interpolación de las coordenadas para evitar errores asociados con longitudes antropométricas incorrectas. Tanto para el suavizado como para la interpolación se utilizarán técnicas basadas en splines de quinto grado desarrollados por WOOD y JENNINGS ${ }^{17}$. En una tercera fase los datos correspondientes a las coordenadas planas (2D) de cada una de las 2 imágenes serán requeridos para la reconstrucción espacial de las coordenadas 3D que definen la columna vertebral, utilizándose para ello la técnica de transformación lineal directa (DLT) descrita por ABDEL-AZIZ y KARARA ${ }^{1}$. Para el correcto funcionamiento de estas técnicas será imprescindible la utilización de la información procedente del objeto de referencia. Tras todo este proceso de transformación se obtendrá una base de datos definitiva que contendrá la información que define la posición espacial (3D) de la columna vertebral, a partir de la cual se iniciará la fase de generación de resultados.

Este proceso de transformación de las coordenadas forma parte de la metodología habitual en el ámbito de la biomecánica deportiva $\left(\mathrm{SOTO}^{13}\right)$, habiéndose adaptado ésta al ámbito específico del estudio de la columna vertebral humana.

\section{Algoritmos de representación gráfica 3D}

Para la generación de resultados se ha elaborado un módulo que permite la creación de representaciones gráficas 3D que simulan de forma realista la columna vertebral humana. Para ello se ha incluido dentro de este módulo un gestor gráfico que permite visualizar diferentes tipos de representaciones gráficas del raquis, desde la simple representación alámbrica, pasando por representaciones que simulan el volumen segmentario (formas geométricas regulares tales como prismas rectangulares, prismas octogonales con apófisis simuladas), hasta llegar a la complejidad de una representación gráfica tridimensional (3D) que simula de forma realista la morfología de las vértebras.

El modelado realista de la columna vertebral ha requerido el desarrollo de algoritmos que permiten obtener la orientación, posición y escalado correcto de los cuerpos vertebrales; esta información ha sido deducida indirectamente a partir de las coordenadas 3D del centro geométrico de los discos intervertebrales. También se han imple- 

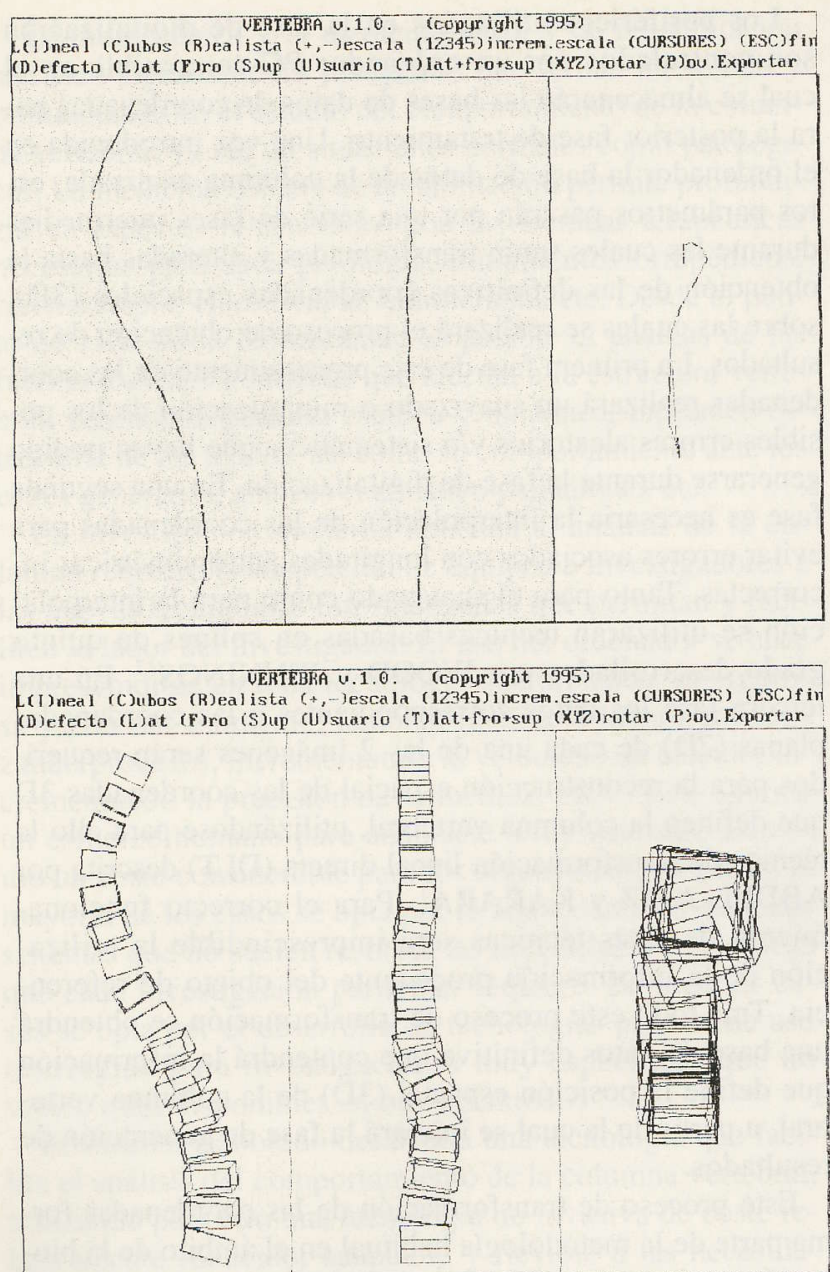

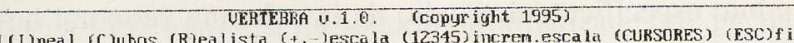
(I)neal (C) ubos (R)ealista (+, - lescala (12345) increm. escala (CURSORES) (ESC)f
(D)efecto (C)at (F)ro (S)up (U) suario (T) lat+fro*sup (XYZ) rotar (P)ou. Exportar

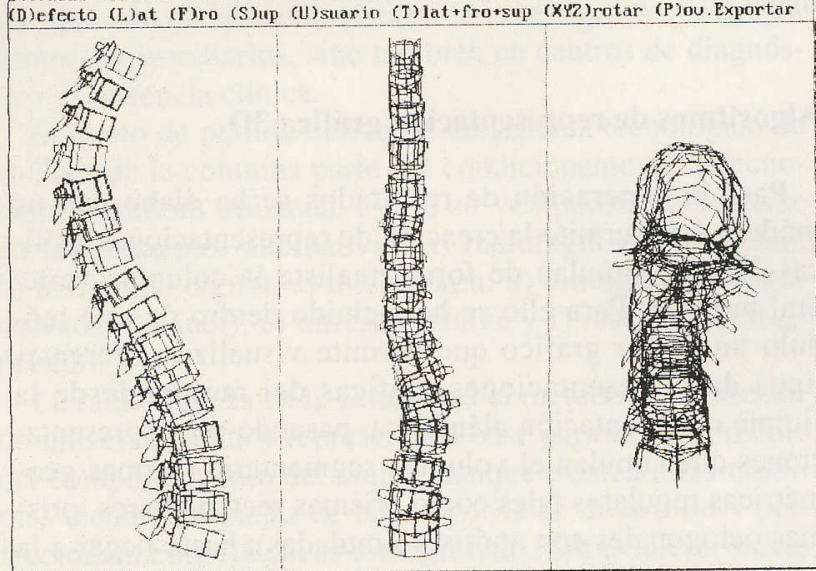

FIG. 2-Modelos de representaciones alámbricas obtenidos.

mentado algoritmos que permiten la creación de imágenes gráficas realistas del modelo humano utilizando eliminación de facetas ocultas y sombreado (rendering). Todo este soporte lógico es gestionado desde una plataforma PCcompatible.
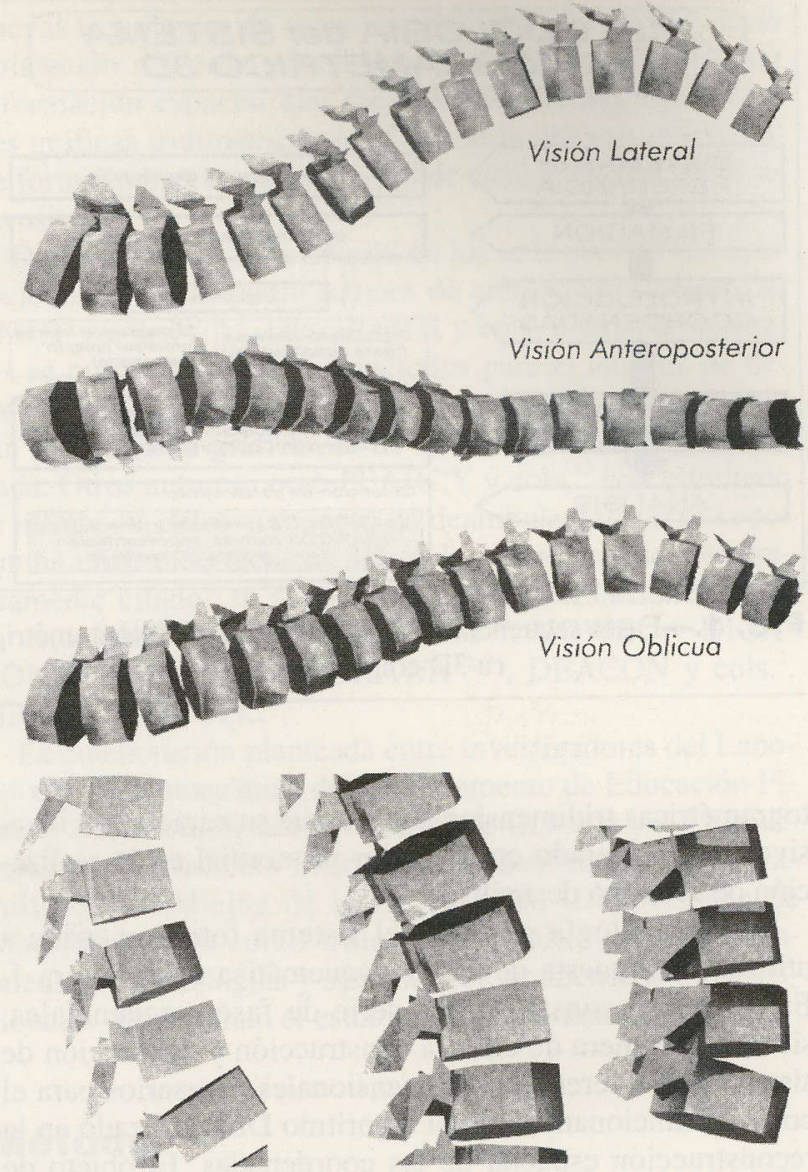

FIG. 3.-Modelos de representaciones renderizadas obtenidos.

\section{Resultados}

El sistema elaborado ha sido aplicado en el estudio de sujetos con escoliosis, llevándose a cabo su validación y puesta a punto. En las figuras 2 y 3 se exponen algunas de las imágenes obtenidas.

\section{Conclusiones}

El sistema elaborado desarrolla una tecnología que facilita el análisis del comportamiento de la columna vertebral, utilizando para ello una tecnología no invasiva de coste relativamente reducido, adaptable y flexible a las necesidades de diversos tipos de situaciones, permitiendo no sólo la investigación básica en centros universitarios, sino también en centros de diagnóstico y asistencia clínica.

En estos momentos la línea de trabajo a seguir se está centrando en la elaboración de un módulo de cálculo que amplíe los resultados del análisis de forma que se pueda incrementar la gama de parámetros numéricos que cuantifiquen y permitan el análisis cuantitativo de la columna analizada, lo cual facilitará la utilización de herramientas estadísticas. Entre estos parámetros específicos destacan los siguientes: 
- Desplazamientos lineales entre cuerpos vertebrales, entre apófisis espinosas, tamaño de los discos intervertebrales, longitud de sectores específicos de la columna, etc.

- Desplazamientos angulares como el ángulo de Perdriolle, detección de las angulaciones máximas y mínimas de inclinación de los cuerpos vertebrales desde un plano sagital, frontal y superior, análisis comparado entre ángulos de orientación de diferentes vértebras.

- Centros de gravedad de cada vértebra individual y del conjunto del raquis humano completo o parcial por sectores.

- Posiciones absolutas y relativas entre vértebras y entre sectores del raquis.

- Otros parámetros específicos para el análisis de determinadas patologías.

\section{Bibliografía}

1. Abdel-Aziz YI, Karara HM. Direct linear transformation from comparator coordinates into object space coordinates in close-range photogrammetry. ASP Symposium on Close Range Photogrammetry. American Society of Photogrammetry. Falls Church, 1971.

2. Deacon P, Flood BM, Dickson RA. Idiopathic scoliosis in three dimensions: a radiographic and morphometric analysis. Journal Bone Joint Surgerv. 1984;66B,4:509-512.

3. De Giorgi G, Gentile A, Paciulli F. Analisi tridimensionale computerizzata del rachide applicata alla scoliosi. Edizione Minerva Medica, 1981.

4. De Smet A, Tarltton M, Cook L, Fritz S, Dwyer S. A radiographic method for three dimensional analysis of spinal configuration. Radiology. 1980;137:343-348.
5. De Smet A, Goin JE, Asher M, Scheuch H. A clinical study of the differences between the scoliotic angles measurement on posteroanterior and anteroposterior radiographs. J Bone Joint Surgery. 1982;64B, 4:490-499.

6. De Smet A, Tarltton M, Cook L, Berridge A, Asher M. The top view for analysis of scoliotic progression. Radiology. 1983;147: 369-372.

7. De Smet A, Asher M, Cook L, Goin J, Scheuch H, Orrick J. Three dimensional analysis of right toracic idiopathic scoliosis. Spine. 1984;9,4:377-381.

8. Graft H, Hecquet J, Dubousset J. Approche tridimensionelle des deformations rachidiennes: application a l'etude du pronostic des scolioses infantiles. Revue de Chirurgie Orthopedique. 1983;69: 407-416.

9. Howel FR, Dickson RA. The deformity of idiopathic scoliosis madfe visible by computer graphics. J Bone Joint Surg. 1989; 71B:399-403.

10. Koyima T, Kurokawa T. Rotation vector: a new method for representation of three dimensional deformity in scoliosis. Spine. 1992a; 17,11:1296-1303.

11. Koyima T, Kurokawa T. Quantification of three dimensional deformity of idiopathic scoliosis. Spine. 1992b;17,35:822-829.

12. Pearcy M, Portek I, Shepherd J. Three dimensional X-ray analysis of normal movement in the lumbar spine. Spine. 1984;11,1:98100.

13. Soto VM. Desarrollo de un sistema para el análisis biomecánico tridimensional del deporte y la representación gráfica realista del cuerpo humano. Edición en microfichas. Tesis Doctoral. Servicio de publicaciones de la Universidad de Granada, 1995.

14. Stokes IA, Bigalow L, Moreland M. Three dimensional spina curvature in idiopathic scoliosis. Journal of Orthopaedic Research. 1987;5:102-113.

15. Stokes IA. Three dimensional terminology of spinal deformity. Spine. 1994; 19,2:236-248.

16. Stokes IA, Bigalow L, Moreland M. Measurement of axial rotation of vertebrae in scoliosis. Spine. 1995;3:212-218.

17. Wood GA, Jennings LS. On the use of spline functions for data smoothing. Journal of Biomechanics. 1979;12:477-479 